medgen $2017 \cdot 29: 239-247$

DOI 10.1007/s11825-017-0135-5

Online publiziert: 4. Juli 2017

(c) Der/die Autor(en) 2017. Dieser Artikel ist

eine Open-Access-Publikation.

CrossMark

\author{
Marion Mutter ${ }^{1}$ Boris Benkner' $\cdot$ Thomas A. Münch ${ }^{1,2}$ \\ ${ }^{1}$ Retinal Circuits and Optogenetics, Centre for Integrative Neuroscience and Bernstein Center for \\ Computational Neuroscience, Universität Tübingen, Tübingen, Deutschland \\ ${ }^{2}$ Institute for Ophthalmic Research, Department of Ophthalmology, Universität Tübingen, Tübingen, \\ Deutschland
}

\title{
Optogenetik als mögliche Therapie bei degenerativen Netzhauterkrankungen
}

attraktiven Kandidaten zur Wiederherstellung der Sehfähigkeit in degenerierter Netzhaut.

In der gesunden Netzhaut findet die Umwandlung von Licht in elektrische Signale in den Photorezeptoren (Stäbchen und Zapfen) statt (- Abb. 1a). Die Absorption von Photonen durch die Stäbchen- und Zapfen-Opsine hat die Aktivierung einer Signalkaskade zur Folge, die letztendlich zur Schließung von Ionenkanälen und somit zur Hyperpolarisation der Photorezeptoren führt. Ein Defekt in diesem Mechanismus kann im schlimmsten Fall komplette Blindheit und Degeneration der Netzhaut verursachen. Viele Zellen der Netzhaut bleiben trotz Photorezeptordegeneration erhalten, und auch die Verknüpfungen zwischen den überlebenden Zellen bleiben für einige Zeit bestehen. Die verbleibenden Zellen können also als Zielzellen zur Expression optogenetischer Proteine genutzt werden. Die Netzhaut würde so ihre Lichtempfindlichkeit wiedererlangen, und den behandelten Patienten sollte auf diese Art und Weise ihre Sehfähigkeit zumindest teilweise wiedergegeben werden können.

In diesem Artikel fassen wir den gegenwärtigen Stand zusammen, wie die Optogenetik als Therapie bei degenerativen Netzhauterkrankungen genutzt werden könnte. Wir beschreiben die wichtigsten Arbeiten der Grundlagenforschungen, die gezeigt haben, dass dieser Ansatz grundsätzlich möglich ist. Wir diskutieren die jeweiligen Vor- und Nachteile der unterschiedlichen Ziel- zellen innerhalb der Netzhaut sowie der bisher genutzten optogenetischen Konstrukte.

\section{Frühe Machbarkeitsstudien}

Schon sehrbald nach derEntdeckungvon Channelrhodopsin erschienen mehrere Studien, die im Mausmodell (i. d. R. mit $r d 1$-Mäusen, deren Photorezeptoren aufgrund einer Mutation degenerieren) das Prinzip bestätigten, dass mittels Optogenetik Blindheit behandelt werden kann. Bereits 2006, nur ein Jahr nach der ersten Veröffentlichung zur Verwendung von ChR-2 in Nervenzellen, gelang es einem Team um Zhuo-Hua Pan aus Detroit erstmals, in degenerierter Netzhaut visuelle Informationsübertragung mithilfe von ChR-2 nachzuweisen [1]. In diesem Ansatz wurde das ChR-2 Gen mithilfe von viralem Gentransfer (mittels adenoassoziierter Viren, AAV) in die Netzhaut von Mäusen eingebracht. Es zeigte sich, dass hier aus technischen Gründen ChR2 überwiegend in den Ganglienzellen exprimiert wurde (• Abb. 1c, d, Ansatz 2a). Diese Ganglienzellen waren somit in der Lage, auf Lichtreize zu reagieren. Der Vorteil einer Expression in den Ganglienzellen, also in der letzten Verarbeitungsschicht der Netzhaut, besteht prinzipiell darin, dass diese auch nach fortgeschrittenem Degenerationsprozess noch lange funktionell erhalten bleiben und in direktem Kontakt zu den visuellen Hirnarealen stehen. Der Nachteil ist, dass die den Ganglienzellen vorgelagerten komplexen Signalverarbeitungsschritte in der beeinflussen $\mathrm{zu}$ können, macht sie $\mathrm{zu}$ 


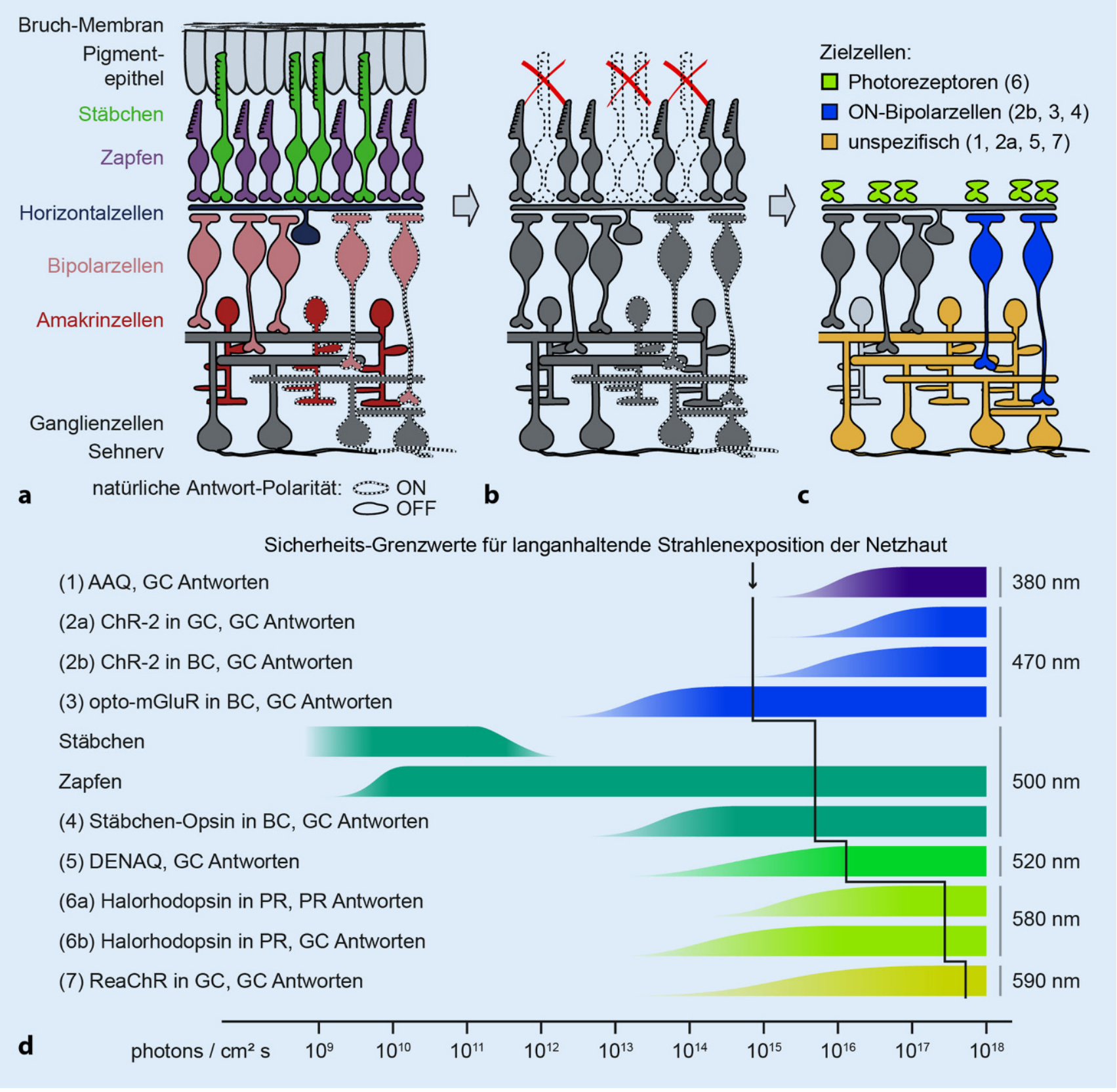

Abb. 1 ॥ Netzhautdegeneration und optogenetische Therapiestrategien $(\mathbf{a}-\mathbf{c})$. a In der gesunden Netzhaut wandeln die Photorezeptoren (Stäbchen und Zapfen) Licht in elektrische Signale um. Ein Teil der nachgeschalteten Zellen wird bei Licht aktiviert (ON-Zellen, gestrichelte Zellumrandung), ein anderer Teil bei Schatten (OFF-Zellen). b Netzhautdegeneration wird oftmals durch Mutationen in den Stäbchen-Photorezeptoren verursacht, die dann als Erstes absterben. c Sekundär verkümmern dann auch die Zapfen. Die im Text beschriebenen optogenetischen Therapien targetieren entweder die Reste der Photorezeptoren (grün) oder die ON-Bipolarzellen (blau), für die man Promotoren zur spezifischen Genexpression kennt. Einige Ansätze sind unspezifisch (orange) und beeinflussen hauptsächlich Ganglienzellen und Amakrinzellen. $d$ Notwendige Lichtintensitäten, um bei den verschiedenen im Text beschriebenen Ansätzen Antworten in der Netzhaut auszulösen. Die verwendeten Konstrukte haben i.d. R. ein breites Anregungsspektrum. Die genannten Wellenlängen entsprechen dem Optimalwert. Der Sicherheitsgrenzwert (schwarze Linie) ist wellenlängenabhängig und entspricht der Europäischen Richtlinie 2006/25/EC. Die notwendigen Lichtintensitäten sind den folgenden Publikationen entnommen: (1) [12], (2a) [1], (2b) [9], (3) [16], (4) [4], (5) [15], (6) [2], (7) [13]. (Abkürzungen: PR Photorezeptoren, BC Bipolarzellen, GC Ganglienzellen) 
Retina verloren gehen, wie zum Beispiel Information über Farbe, Kontrast oder Bewegung.

Dies war einer der Gründe für einen neuartigen Ansatz, der 2008 von Botond Roska und seinem Team aus Basel vorgestellt wurde [9]. Um neben der Lichtsensitivität auch weitere Verarbeitungseigenschaften der Netzhaut wiederherzustellen, brachten sie ChR-2 spezifisch in ON-Bipolarzellen ein (• Abb. 1c, d, Ansatz 2b). In der gesunden Netzhaut gibt es parallele Wege der Informationsverarbeitung, so werden zum Beispiel ONBipolarzellen während eines Lichtreizes depolarisiert, während OFF-Bipolarzellen hyperpolarisiert werden (• Abb. 1a). ChR-2 in ON-Bipolarzellen führt also zu deren natürlicher Reaktion auf Licht, nämlich zur Depolarisation. Diese Eigenschaft wurde auf die nachgeschalteten ON-Ganglienzellen übertragen, während für OFF-Ganglienzellen keine Antworten nachgewiesen werden konnten. Später zeigte sich, dass bei ausreichender Expressionsrate auch OFF-Ganglienzellen aktiviert werden, und zwar richtigerweise nach dem Ende eines Lichtreizes. Zusätzlich konnten auch rezeptive Feldeigenschaften nachgewiesen werden, wie etwa die center-surround Verarbeitung, und die unterschiedlichen Antwortdynamiken verschiedener Zellen mit phasischen und tonischen Antworten. Erstmals wurde hier auch bei den so behandelten blinden Tieren visuell ausgelöstes Verhalten nachgewiesen.

In einem nächsten Schritt konnte 2010 gezeigt werden, dass sich auch Photorezeptoren als Zielzellen eignen. Während die Stäbchen-Photorezeptoren (abhängig vom spezifischen genetischen Defekt) sehr früh absterben, bleiben Zapfen-Photorezeptoren länger erhalten (• Abb. 1c; [2]). Obwohl auch Zapfen das Außensegment und ihre Lichtempfindlichkeit verlieren, können sie weiterhin eingebrachte optogenetische Gene exprimieren. Darauf aufbauend brachte Volker Busskamp aus der Arbeitsgruppe von Botond Roska (2010) Halorhodopsin in noch übrig gebliebene Zellkörper von Zapfen ein (•Abb. 1c, d, Ansatz 6). Da Halorhodopsin durch Lichtaktivierung zur Hyperpolarisation von Zellen führt, konnte somit die natürliche Re-

medgen 2017· 29:239-247 DOI 10.1007/s11825-017-0135-5

๑ Der/die Autor(en) 2017. Dieser Artikel ist eine Open-Access-Publikation.

M. Mutter · B. Benkner · T. A. Münch

\section{Optogenetik als mögliche Therapie bei degenerativen Netzhauterkrankungen}

\section{Zusammenfassung}

Bei neurodegenerativen Erkrankungen der Netzhaut sind die lichtempfindlichen Zellen, die Photorezeptoren, oft als Erstes betroffen. Die Optogenetik ist ein vielversprechender Ansatz, die Netzhaut wieder lichtempfindlich zu machen und dadurch das Sehvermögen wiederherzustellen. Bei der Optogenetik werden lichtempfindliche Proteine über gentechnische Methoden in die Netzhaut eingebracht; die Aktivität der Zielzellen wird durch diese Behandlung durch Licht beeinflussbar. Dieser Einfluss kann die direkte lichtinduzierte Änderung des Membranpotenzials sein (sowohl hemmend als auch erregend) oder die lichtinduzierte Aktivierung intrazellulärer Signalkaskaden. Dies hat zur Folge, dass das Zielgewebe, die Netzhaut, wieder auf Licht reagiert. Diese Übersicht beschreibt die Prinzipien der Optogenetik und den gegenwärtigen Stand im Hinblick auf ihre Anwendung zur Behandlung von Blindheit.

Schlüsselwörter

Optogenetik - Chemische Photoswitches . Netzhautdegeneration - Channelrhodopsin . Retina

\section{Optogenetics as a possible treatment for degenerative retinal diseases}

\section{Abstract}

The light-sensitive cells of the retina, the photoreceptors, are often the first cells affected in neurodegenerative diseases of the eye. Optogenetics is a promising approach in which the retina is re-sensitized and the visual ability is restored. In optogenetics, light-sensitive proteins are expressed in the retina with the help of gene transfer; the activity of the target cells can then be influenced by light. This influence may be in the form of direct light-induced alterations of the membrane potential (either inhibitory or excitatory), or through the light-induced activation of intracellular signaling cascades. As a result, the target tissue, i. e., the retina, can react to light again. This review describes the principles and current state of optogenetic approaches to the treatment of blindness.

Keywords

Optogenetics - Chemical photoswitches . Retinal degeneration · Channelrhodopsin . Retina aktion von Photorezeptoren auf Licht durch optogenetische Methoden ersetzt werden. Die Folge war eine nochmals verbesserte Restoration der retinalen Informationsverarbeitung. Weiterhin war dies die erste Studie, die diesen Ansatz auch in Kulturen mit menschlicher Netzhaut anwendete und zeigte, dass die Expression dort prinzipiell möglich ist.

\section{Optimierung optogenetischer Konstrukte für visuelle Restoration}

Diese Machbarkeitsstudien haben große Hoffnungen geweckt, mit der Optogenetik einen neuen, sehr effizienten Therapieansatz zur Behandlung von degenerativen Netzhauterkrankungen gefunden zu haben. Gleichzeitig wurden zwei große Defizite der klassischen optogenetischen Proteine ChR-2 und Halorhodopsin offensichtlich, nämlich die zu ihrer Aktivierung nötigen hohen Lichtintensitäten und damit verbunden die nicht vorhandene Adaptation an unterschiedliche Lichtverhältnisse. Dies ist bei grundlagenwissenschaftlichen Anwendungen der Optogenetik im Labor kein Problem, hier wird das entsprechend behandelte Gewebe mit Laserlicht oder entsprechend hellen LEDs angeregt. Zur Therapie von Blindheit wäre es allerdings wünschenswert, dass normales Umgebungslicht ausreicht, um die optogenetischen Proteine anzuregen. 
Die Lichtintensitäten, die in den hier beschriebenen Studien zur Aktivierung der Netzhaut nötig waren, sind in - Abb. 1d zusammenfassend dargestellt. Für ChR2 und Halorhodopsin wäre dies nur in äußerst hellen Umgebungen der Fall, zum Beispiel während des Aufenthaltes auf einem sonnenbeschienenen Gletscher. Ein hell eingestellter Fernseher oder ein Computermonitor erzeugen dagegen nicht genügend Licht.

\section{Technische Unterstützungs- maßnahmen}

Als eine mögliche Lösung für dieses Problem wird an der Entwicklung von „virtual reality“ (VR) Brillen gearbeitet, die als Display ein Array aus sehr hellen LEDs benutzen. Auf diesem Display wird die Umgebung gezeigt, die von einer integrierten Kamera erfasst wird. Ein Vorteil dieses Systems besteht darin, dass die benutzten LEDs und die benötigte Helligkeit an das verwendete optogenetische Protein angepasst werden können. ChR-2 zum Beispiel hat seine maximale Empfindlichkeit gegenüber blauem Licht (ca. $470 \mathrm{~nm}$ ), Halorhodopsin gegenüber gelbem Licht (ca. $580 \mathrm{~nm}$ ). Unabhängig von der tatsächlichen Umgebungshelligkeit kann damit immer die optimale Anregung des therapeutischen Optogens gewährleistet werden. Außerdem lässt sich im Prinzip jede gewünschte Bildverarbeitung auf das Kamerabild anwenden, um den Seheindruck des Patienten zu verbessern. Die Verstärkung von Kontrasten im Bild könnte zum Beispiel das Erkennen von Objekten vereinfachen [18]. Allerdings umgeht dieser Ansatz nicht das Problem, dass die benötigten hohen Lichtintensitäten, insbesondere bei Wellenlängen im Bereich von 400-500 nm, auf Dauer schädlich für das Gewebe sind $[7,13]$.

\section{Optimierte Varianten von Channelrhodopsin}

Eine zweite Lösung des Problems ist daher die Nutzung von optogenetischen Proteinen, die das vorhandene Licht effizienter in Anregung des Gewebes umsetzen. Drei Faktoren bestimmen diese Effizienz und damit die effektive
Lichtempfindlichkeit, nämlich die Expressionseffizienz, die Wahl der Zielzelle und Art der Beeinflussung der Zielzelle. Die Expressionseffizienz beeinflusst schlicht die Anzahl der vorhandenen Proteine. Eine Verdopplung der Anzahl von ChR-2 Proteinen in einer Zelle verdoppelt die Wahrscheinlichkeit, dass eines davon bei gegebener Lichtstärke ein Photon absorbiert. Da ChR-2 ein Membranprotein ist, ist eine Erhöhung der Anzahl allerdings durch die Oberfläche der Zelle beschränkt, und eine Überfrachtung der Zellmembran mit einem Fremdprotein hat negative Auswirkungen auf die Gesundheit einer Zelle. Außerdem benötigen die gegenwärtigen Ansätze nicht eine Verdoppelung der Lichtempfindlichkeit, sondern eine 1000-fache Erhöhung. Nichtsdestotrotz, die Evolution zeigt uns, dass dies im Prinzip eine sehr gewinnbringende Strategie wäre, schließlich sind die Außensegmente der Photorezeptoren genau aus diesem Grund mit Membranstapeln, den discs, vollgepackt.

Der zweite Faktor ist die Zielzelle für die optogenetische Behandlung. Durch Konvergenzeffekte innerhalb des retinalen Netzwerks steigt die effektive Lichtempfindlichkeit, wenn das optogenetische Protein mehr distal in der Netzhaut exprimiert wird. Dies ist besonders eindrücklich bei Expression in Photorezeptoren (-Abb. 1c, d, Ansatz 6). Bei direkter Ableitung von den Photorezeptoren selbst konnten Antworten erst bei sehr hohen Lichtintensitäten festgestellt werden (Ansatz 6a). Durch Pooling des schwachen Photorezeptorsignals zeigten Ganglienzellen allerdings schon robuste Antworten bei deutlich geringeren Intensitäten (Ansatz 6b). Ein ähnlicher Effekt ist sichtbar beim Vergleich von ChR-2 Expression in Bipolarzellen (Ansatz 2b) vs. Ganglienzellen (Ansatz 2a).

Der dritte Faktor ist die Auswirkung auf die Zelle, wenn ein Photon absorbiert worden ist. Im Gegensatz zu Photorezeptoren, in denen das Rhodopsin über den Umweg einer Signalkaskade die Öffnung eines Ionenkanals beeinflusst, ist ChR-2 selbst ein Ionenkanal. Nach der Aktivierung durch ein absorbiertes Photon öffnet sich dieser Kanal und bleibt für eine bestimmte Zeit offen.
Die Länge dieser Öffnungszeit ist eine Stellschraube zur Regulierung bei ChR2: Je länger anhaltend die Öffnung des Kanals, desto größer ist die Auswirkung auf das Membranpotenzial der Zelle. Allerdings ist diese Öffnungszeit eine inhärente Eigenschaft des Proteins selbst. Es gibt verschiedene Varianten von Channelrhodopsin, bei denen diese Zeit kürzer oder länger ist als beim ursprünglich beschriebenen ChR-2 (von wenigen Millisekunden bis viele Minuten). Zur Erhöhung der Effizienz eignen sich zur Therapie im Auge also eher die Varianten, die eine etwas längere Öffnungszeit haben, ohne jedoch zu träge auf Lichtänderungen zu reagieren. Eine zweite inhärente Kanaleigenschaft, die sich auf die effektive Lichtempfindlichkeit auswirkt, ist die Permeabilität gegenüber bestimmten Ionen. So hat die Channelrhodopsin-Variante CatCh eine erhöhte Permeabilität für Kalziumionen; erhöhter Kalziumeinstrom verstärkt mittels sekundärer Effekte die Reaktion der Zelle auf den Lichtreiz [8]. Diese beiden Ansätze - Öffnungszeit und Ionenspezifizität - reichen aber immer noch nicht aus, die gewünschte Sensitivität $\mathrm{zu}$ erzielen. Eine dritte inhärente Eigenschaft ist die spektrale Empfindlichkeit. ChR-2 selbst hat seine höchste Empfindlichkeit im Blaubereich (470 nm). Solch kurzwelliges Licht führt aber schnell zu phototoxischen Effekten auf das bestrahlte Gewebe [17]; langwelligeres Licht hat einen deutlich höheren Gefahrengrenzwert (• Abb. 1d, schwarze Linie). Channelrhodopsine mit rotverschobenem Spektrum eignen sich daher eher zur Therapie. Zwar führt dies nicht an sich $\mathrm{zu}$ einer erhöhten Sensitivität, allerdings sind die benötigten hohen Lichtintensitäten weniger schädlich für das Auge. Die Arbeitsgruppen um Deniz Dalkara und Jens Dübel in Paris haben kürzlich die Eignung der Channelrhodopsinvariante ReaChR untersucht (• Abb. 1c, d, Ansatz 7; [13]). Deren Anregung war mit Lichtintensitäten möglich, die sich zwar nicht sehr von ChR-2 unterschieden, aber dennoch deutlich unterhalb des im Rotbereich höheren Sicherheitsgrenzwertes lagen. Außerdem konnten sie zeigen, dass sich ReaChR in Primatenretina ex- 
primieren und anregen lässt, inklusive menschlicher Netzhaut.

\section{Nutzung intrazellulärer Signalkaskaden}

Eine weitere Art der Zellbeeinflussung besteht darin, dass man sich vorhandene Signalkaskaden in der Netzhaut zunutze macht und dafür optogenetische Alternativen zu ChR-2 entwickelt, die sich speziell für Therapieansätze in der Netzhaut eignen. Vorbild sind hier die gesunden Photorezeptoren, bei denen jedes aktivierte Rhodopsin eine Signalkaskade anstößt, die relativ lang anhaltend zur Schließung von Membrankanälen und letztlich zur robusten Hyperpolarisation des Photorezeptors führt. Die Absorption des Photons ist also nur der Auslöser der Signalkaskade. Eine Signalkaskade mit mehreren Schritten hat einen weiteren Vorteil: Viele Schritte bedeuten viele Stellschrauben, mit denen die Gesamtverstärkung des Signals reguliert werden kann. Diese Adaptation ist der Hauptgrund dafür, dass die gesunde Netzhaut über einen riesigen Helligkeitsbereich hinweg funktioniert. Auch bei optogenetischen Ansätzen führt das Andocken an existierende Signalkaskaden zu Signalverstärkung. Außerdem greifen automatisch die vorhandenen Regulierungsmechanismen der Kaskade, sodass ein gewisses Maß an Adaptation erzielt wird. Zwei Beispiele sollen hier vorgestellt werden.

Das Team um Rob Lucas in Manchester und, unabhängig davon, ein Team um John Flannery aus Berkeley, exprimierten das Stäbchen-Rhodopsin ektopisch in nachgeschalteten Verarbeitungsschichten degenerierter Netzhaut, wodurch erfolgreich Lichtantworten erzeugt wurden (• Abb. 1d, Ansatz 4; [4, 6]). Offensichtlich kann sich Rhodopsin auch in anderen Zellen, nicht nur in den Photorezeptoren, in G-Proteingekoppelte Signalkaskaden einklinken und lichtgesteuerte Antworten auslösen. Es ist bekannt, dass Rhodopsin aufgrund des Ausbleichens grundsätzlich Nachschub an Retinal (das Licht absorbierende Chromophor des Opsins) benötigt, was normalerweise über den engen Kontakt zwischen den Photore- zeptoren und der Pigmentepithelschicht gewährleistet wird. Überraschend war, dass die Antworten ohne externe $\mathrm{Zu}$ gabe von Retinal in vivo über längere Zeit stabil blieben. Das in der Netzhaut dieser Degenerationsmauslinie noch zur Verfügung stehende Retinal reichte also offensichtlich zur Regeneration aus. Allerdings sind diese Ergebnisse nicht ausreichend, um Rückschlüsse auf den Therapieerfolg beim Menschen zuzulassen. Das Problem des Ausbleichens bleibt insoweit ungeklärt, und explizite Studien zur Regeneration des Rhodopsin in dieser Therapievariante werden nötig sein. In diesen Studien wurden zwei verschiedene Expressionsstrategien in rd1-Mäusen verwendet, zum einen eine unspezifische Expression in allen Zelltypen der Netzhaut (hierbei wurden überwiegend die inneren Schichten der Netzhaut erreicht), zum anderen eine ON-bipolarzellspezifische Expression. Beide Herangehensweisen führten sowohl in den Ganglienzellen als auch im Thalamus zu einer Wiederherstellung der Lichtantworten. Verhaltensversuche zeigten, dass die spezifische Expression in ON-Bipolarzellen einen Seheindruck mit besserem räumlichen Auflösungsvermögen ermöglichte.

Die Arbeitsgruppe von Sonja Kleinlogel in Bern hat ein komplett neuartiges optogenetisches Konstrukt entwickelt, Opto-mGluR, das sich die Signalkaskade der ON-Bipolarzellen zunutze macht [16]. Diese Kaskade wird normalerweise vom Glutamatrezeptor mGluR6 aktiviert, der auf das von Photorezeptoren ausgeschüttete Glutamat reagiert. Das neu entwickelte Opto-mGluR ist ein chimäres Membranprotein, dessen intrazellulärer Anteil aus den entsprechenden Peptidsequenzen von mGluR6 besteht und daher an die Signalkaskade andocken kann. Der Rest des Proteins besteht aus den entsprechenden Peptidsequenzen von Melanopsin, ein natürlicherweise in der Netzhaut vorkommendes Opsin. Lichtaktivierung des Melanopsinanteils führt nun in der Tat zu einer Aktivierung der Signalkaskade. Zwar ist das Absorptionsspektrum von Melanopsin, vergleichbar mit ChR-2, im blauen Bereich, allerdings reichte hier bereits eine 1000-fach geringere Aktivierungs- energie (• Abb. 1c, d, Ansatz 3). Vermutlich liegt diese erhöhte Effizienz tatsächlich daran, dass Opto-mGluR die G-protein gekoppelte Signalkaskade in ON-Bipolarzellen aktiviert, die zu einer deutlichen Verstärkung des Signals führt. Interessanterweise führt die Aktivierung des Opto-mGluR Proteins zu invertierten Signaleigenschaften in ON-Bipolarzellen im Vergleich zum normalen $\mathrm{Zu}$ stand. Die Kaskade wird mittels OptomGluR durch Licht aktiviert. In der gesunden Netzhaut hingegen ist die Kaskade bei Dunkelheit aktiv (aufgrund des dann hohen Glutamatausstoßes der Photorezeptoren) und wird durch Licht abgeschaltet. Nichtsdestotrotz erreichte man mithilfe von Opto-mGluR die Wiederherstellung der Sehleistung in der Retina, bei der (wenn auch invertiert) sowohl ON- als auch OFF-Antworten abgeleitet werden konnten. Darüber hinaus waren die Lichtantworten auch in kortikalen Arealen (V1) zu finden. Die behandelten Mäuse zeigten sowohl den optomotorischen Reflex als auch Lernverhalten auf visuelle Stimuli im Schwimmtank. Bei diesen Verhaltensversuchen waren moderate Helligkeiten ausreichend, um visuell gesteuertes Verhalten auszulösen.

In beiden Studiendesigns wurden lichtempfindliche Proteine verwendet, die natürlicherweise in der Netzhaut vorkommen (Rhodopsin) oder aus natürlich vorkommenden Proteinanteilen zusammengesetzt sind (Melanopsin bei Opto-mGluR). Gerade hinsichtlich zukünftiger klinischer Studien und Anwendungen ist dies sehr von Vorteil, da das Risiko von Immunreaktionen reduziert ist. Beide Ansätze konnten das Problem der übermäßig hohen Lichtintensitäten überwinden und erweitern durch die Ankopplung an G-proteinKaskaden die Adaptationsfähigkeit. So wurde die Empfindlichkeitsbreite von 2-3 (bei ChR-2) auf 4-6 Größenordnungen ausgedehnt. Allerdings ist die Anpassungsbreite damit immer noch ein Stück von der natürlichen entfernt.

\section{Eine Alternative: Photopharmakologie}

Ein verwandter, dennoch grundsätzlich anderer Ansatz zur Wiedererlangung der 
Lichtempfindlichkeit bei Netzhautdegeneration liegt in der Nutzung sogenannter chemischer Photoswitches. Hierbei handelt es sich um kleine Moleküle, die nach Absorption eines Photons ihre Konfiguration ändern. Insofern verhalten sich diese Moleküle nicht grundsätzlich anders als Retinal, das Chromophor der Opsine, das durch seine Konformationsänderung das Opsin (Rhodopsin oder (hR-2) in den angeregten Zustand überführt. Bei der Photopharmakologie nutzt man nun Moleküle, die in der passenden (lichtaktivierten) Konformation als Ligand dienen, etwa für Glutamatoder GABA-Rezeptoren, und dadurch die Aktivierung oder Hemmung von Zellen verursachen. Alternativ können auch Moleküle eingesetzt werden, die in entsprechender Konformation als spezifische Blocker für Ionenkanäle agieren. Grundsätzlich werden diese Moleküle ins Auge injiziert und lösen dann in der Netzhaut durch Anlagerung an die vorhandenen Rezeptoren bzw. Kanäle lichtinduzierte Aktivität aus. Solche photopharmakologischen Ansätze teilen sich mit der Optogenetik also die Eigenschaft, dass nach der Behandlung die Aktivität der Netzhaut direkt mittels Licht beeinflusst werden kann. Wir beschreiben hier daher erste Studien hierzu, obwohl die Photopharmakologie aufgrund der fehlenden genetischen Komponente nicht in die engere Kategorie der optogenetischen Therapien fällt.

Ein erster Vertreter dieser Klasse von synthetischen Molekülen, welcher zur Therapie in degenerierter Netzhaut bei Mäusen getestet wurde, war das Acrylamid-Azobenzol-Quaternäre Ammonium, kurz AAQ (• Abb. 1c, d, Ansatz 1) [12]. In seiner trans-Konformation ist AAQ ein effektiver Blocker von Kaliumkanälen, was die Anregung von Neuronen zur Folge hat. Durch UVLicht wird es in cis-AAQ überführt, was die Kanäle wieder öffnet und die Zellen hemmt. Langsame thermische Isomerisation, oder grünes Licht, verursachen wieder trans-AAQ. In der Arbeitsgruppe von Richard Kramer in Berkeley wurde der Effekt von AAQ auf die Lichtantworten in degenerierter Mausnetzhaut untersucht [12]. Degenerierte Netzhaut von $r d 1$-Mäusen wurde durch Zugabe von AAQ wieder lichtsensitiv. Interessanterweise führte hauptsächlich die Überführung von trans-AAQ zu cisAAQ mit UV-Licht zur Aktivierung von Ganglienzellen, obwohl die cisForm Zellen hemmt. Vermutlich lag dies daran, dass die meisten Kaliumkanäle sich in den Membranen von inhibitorischen Amakrinzellen befinden und deren Hemmung eine effektive Aktivierung der Ganglienzellen bedingte. AAQ war bis zu $5 \mathrm{~h}$ nach Applikation funktional. Auch bei vorheriger Invivo-Injektion des Moleküls ins Auge (12 h vorab) und anschließender elektrophysiologischer Ableitung zeigte die Netzhaut Lichtantworten. Zusätzlich gelang es nach intraokularer Injektion von AAQ, den Pupillenreflex und Lichtvermeidungsverhalten nachzuweisen. Trotz dieser vielversprechenden Resultate eignet sich AAQ wohl nur wenig für klinische Studien, da sich das Molekül sehr schnell im Körper abbaut, sodass tägliche Injektionen nötig wären. Zudem werden hier, wie bei den klassischen optogenetischen Proteinen, sehr hohe kurzwellige Lichtintensitäten benötigt, wobei der optische Apparat des Menschen die eigentlich nötigen (aber schädlichen) UV-Anteile größtenteils herausfiltert.

Ein verwandtes Molekül zweiter Generation, DENAQ (• Abb. 1c, d, Ansatz 5), hat viele der Nachteile von AAQ ausgemerzt [15]. Es hat eine 100-fach höhere Lichtsensitiviät, ist durch weißes Licht erregbar (trans $\rightarrow$ cis) und regeneriert im Dunkeln sehr schnell in den trans-Zustand. Außerdem ist die physiologische Halbwertszeit von DENAQ mit etwas über zwei Tagen deutlich höher als bei AAQ $(3,6 \mathrm{~h})$, sodass wöchentliche Injektionen ausreichen würden. Auch in Verhaltensversuchen, wie dem Open-field-Test und Konditionierungsversuchen, konnte eine lichtinduzierte Verhaltensänderung nachgewiesen werden. Überraschenderweise funktioniert die Generierung von Lichtantworten mithilfe von DENAQ in degenerierter Netzhaut deutlich effizienter als in gesunder Netzhaut, vermutlich weil DENAQ neben Kaliumkanälen hauptsächlich HCN-Kanäle bindet und diese als Folge der Degeneration vermehrt in Ganglienzellen exprimiert werden. Dieser Ansatz stellt eine vielversprechende Möglichkeit für Therapien dar und könnte zukünftig bei bestimmten Netzhauterkrankungen helfen, das Augenlicht wiederherzustellen. Die Tatsache, dass DENAQ und verwandte Moleküle im Auge wieder abgebaut werden, kann durchaus auch ein Vorteil sein: So könnten damit grundsätzlich die Möglichkeiten der Photoaktivierung degenerierter Netzhaut getestet werden, als erster Schritt vor anderen permanent wirkenden Herangehensweisen.

\section{Welche Netzhauterkrankungen eignen sich zur Therapie mit Optogenetik?}

Die Anwendung optogenetischer Therapien ist grundsätzlich unabhängig vom Krankheitsbild und ist in verschiedenen Stadien der Netzhautdegeneration einsetzbar. Allerdings müssen für zukünftige optogenetische Therapien zumindest die Ganglienzellen noch intakt sein. Generell sind Krankheitsbilder besonders im Fokus der optogenetischen Therapie, bei denen das Pigmentepithel oder die Photorezeptoren beeinträchtigt werden. Hierunter fallen beispielsweise die häufigsten Formen von Netzhautdegeneration: die altersabhängige Makuladegeneration (AMD) und Retinitis pigmentosa (RP). Bei der AMD, welche vornehmlich im höheren Alter auftritt, sind primär nichtneuronale Retinastrukturen betroffen. Bei fortschreitender Degenerieren des retinalen Pigmentepithels, der BruchMembran und der Aderhaut hat dies Auswirkungen auf die darunter befindlichen Photorezeptoren in der Makula. AMD betrifft meist zunächst die Zapfen in der zentralen Retina und führt zum Sehverlust des Bereiches des schärfsten Sehens. Mit über 25 Mio. betroffenen Patienten weltweit gehört sie zu den häufigsten stark beeinträchtigenden Sehstörungen. RP hingegen zählt zu den Krankheiten, die bereits recht früh in der Entwicklung einsetzen. Hierbei kommt es durch den Ausfall der peripheren Stäbchen-Photorezeptoren zu einer Verschlechterung des Dunkelsehens. Darauf folgt eine langsame, oft über Jahre hinweg andauernde 
Degeneration der Zapfen-Photorezeptoren, welche zu einem sukzessiven Visusabfall führt. Der Verlust der Zapfen resultiert in einem immer kleiner werdenden Gesichtsfeld, dem Tunnelblick, und endet letztlich in Blindheit. Da sich aufgrund der zahlreichen Mutationen, die mit der Krankheit assoziiert sind, Gentherapie als besonders schwierig erweist, sind hier optogenetische Ansätze eine sinnvolle Alternative.

Grundsätzlich gilt: Je mehr von den verarbeitenden Strukturen der Netzhaut noch vorhanden ist, desto mehr Möglichkeiten bestehen bei der Wahl der geeigneten Zielzelle und des passenden optogenetischen Proteins. Je früher in der retinalen Signalverarbeitung angesetzt werden kann (idealerweise in den funktionell defekten Photorezeptoren oder in den Bipolarzellen), desto mehr der eigentlichen Funktionalität der Retina kann durch die wiedererlangte Lichtsensitivität genutzt werden. Allerdings findet bei fortschreitender Degeneration ein zunehmender Umbau der Netzhaut statt, das heißt die ursprünglichen zellspezifischen Verknüpfungen gehen mehr und mehr verloren [10]. Trotzdem steht die innere Netzhaut weiterhin zur Transmission der Signale zur Verfügung. Bei der nassen Form der AMD zum Beispiel überleben mehr als $50 \%$ der Ganglienzellen, bei trockener AMD fast alle, selbst wenn kaum noch gesunde Photorezeptoren vorhanden sind. Bei RP überleben selbst im Spätstadium $70-80 \%$ der Bipolarzellen und 25-40\% der Ganglienzellen [18].

Aufgrund der guten Zugänglichkeit des betroffenen Gewebes, der Netzhaut, können die optogenetischen Konstrukte relativ einfach und sehr zielgenau appliziert werden. In nahezu allen hier erwähnten Ansätzen der Grundlagenforschung wurden Viren als Genfähren benutzt, um diese durch subretinale oder intravitreale Injektion in die entsprechenden Zellschichten zu transportieren. Abhängig von den jeweiligen Promotoren können so spezifisch Zielzellen erreicht werden. Zum derzeitigen Stand der Forschung ist die Auswahl an retinalen spezifischen Promotoren noch sehr beschränkt. Dies ist mit ein Grund dafür, dass die oben beschrie- benen spezifischen Ansätze sich auf Photorezeptoren und ON-Bipolarzellen beschränken (बAbb. 1c). $\mathrm{Zu}$ den klinisch relevantesten Viren zählen die adenoassoziierten Viren, die bereits in anderen Gentherapiestudien Anwendung finden. Derzeit sind sowohl einige Studien an Primaten wie auch erste klinische Studien angelaufen (RetroSense Therapeutics: NCT02556736; Gensight: zzt. noch vorklinisch), die zunächst die konzentrationsabhängigen Effekte untersuchen, um eine sichere und gut verträgliche Applikationsmenge zu finden. Diese Studien befinden sich jedoch höchstens in Phase 1 und werden vorerst noch mit ChR-2 durchgeführt. Langfristig ist die Verwendung von neu entwickelten Varianten wahrscheinlich, wie etwa Opto-mGluR oder ReaChR, welche mit deutlich niedrigeren Lichtintensitäten aktivierbar sind oder mit Licht im langwelligen Bereich, dessen nötige Intensität weiter vom Sicherheitsgrenzwert entfernt ist ( $\bullet$ Abb. 1d).

\section{Vergleich der Optogenetik und anderer Therapieformen}

Optogenetik ist zwar in jedem Stadium der Degeneration zur Wiederherstellung der Sehfunktion einsetzbar, allerdings wirkt die optogenetische Behandlung der Krankheitsursache nicht entgegen und nimmt somit keinen Einfluss auf den weiteren Verlauf. Grundsätzlich besteht zwar die Möglichkeit, dass die Stimulation des neuronalen Netzwerks einen protektiven Effekt haben und zu einer Verlangsamung der Degeneration führen könnte, allerdings fehlt dafür die Datengrundlage. Bei Anwendung in relativ frühen Stadien der Degeneration, wenn noch distale Verarbeitungsschichten der Netzhaut (z. B. Photorezeptoren) als Zielzellen zur optogenetischen Behandlung zur Verfügung stehen, könnte die zusätzliche Behandlung mit Neuroprotektiva die Überlebensdauer der behandelten Zellen verlängern. Ein Beispiel hierfür ist das Peptid rod-derived cone viability factor (RdCVF), das in degenerierender Netzhaut die Zapfenfunktion verbessert und das Absterben verlangsamt [3].
Generell stellt sich bei optogenetischen Ansätzen die Frage, inwieweit die Plastizität der kortikalen Sehareale ausreicht, um die neuartige und in gewissem Grade unnatürliche Information $\mathrm{zu}$ interpretieren und einen Seheindruck zu generieren. Die Erkenntnisse mit Patienten mit einem implantierten bioelektrischen retinalen Chip zeigen jedoch, dass dies kein größeres Problem sein sollte: Selbst bei sehr fortgeschrittenem Degenerationsverlauf wurden mit elektrischen Netzhautimplantaten Seheindrücke erfolgreich wiederhergestellt [14]. Im Vergleich zum bioelektrischen Chip sollten optogenetische Ansätze mehrere Vorteile bieten. Das zum Gehirn übermittelte Signal sollte hier eher dem natürlichen Netzhautsignal entsprechen. Außerdem bieten optogenetische Ansätze den großen Vorteil, dass sie in der gesamten Netzhaut exprimiert werden können und so insgesamt ein besseres Auflösungsvermögen erwarten lassen. Zudem werden bei optogenetischer Behandlung von Bipolarzellen/ Photorezeptoren auch die Unterschiede zwischen der neuralen Verschaltung in der peripheren und der zentralen Retina widergespiegelt. Ein weiterer Vorteil dürfte die wenig invasive Injektion sein, im Vergleich zum chirurgischen Eingriff bei der Einbringung des bioelektrischen Chips.

Nach wie vor ungelöst ist das Problem der mangelnden Adaptationsfähigkeit. Um auch in dunkleren Umgebungen einen Seheindruck zu erreichen, werden wie oben beschrieben intensitätsverstärkende VR-Brillen zusätzlich zur optogenetischen Behandlung nötig sein. Insgesamt geht sowohl bei optogenetischen Proteinen als auch bei photopharmakologischen Ansätzen der Trend zu langwelligeren Varianten, da das für die Aktivierung benötigte langwellige Licht weniger schädlich ist. Langfristig wäre mit geeigneten Promotoren auch ein gezielter Ansatz bei Farbenblindheit, beziehungsweise die Wiederherstellung von Farbsehen selbst nach vollständiger Erblindung denkbar. Ein wichtiger Punkt für klinische Studien ist zudem die Halbwertszeit der Proteine im Organismus. Chemische Photoswitches haben zunächst den Vorteil, dass sie im Körper relativ schnell 
wieder abgebaut werden und so eventuelle noch ungeklärte Nebenwirkungen nur temporär auftreten. Allerdings ist diese kurze Halbwertszeit im Hinblick auf eine lebenslange Therapie eher nachteilig, da wöchentliche Injektionen nötig wären. Eine Lösung hierfür könnten neu entwickelte intraokuläre Depotsysteme sein, die Wirkstoffe langsam und stetig abgeben.

\section{Fazit für die Praxis}

Zusammenfassend kann gesagt werden, dass sowohl optogenetische Proteine als auch chemische Photoswitches sehr vielversprechend für die Therapie bei Netzhautdegeneration sind. Insbesondere für Channelrhodopsin-basierte Ansätze findet bereits eine Translation zu Studien am Primaten und am Patienten statt. Erste Ergebnisse aus klinischen Studien sind in naher Zukunft zu erwarten. Allerdings sind noch einige Optimierungen nötig, sowohl bei den optogenetischen Proteinen als auch bei den zum Gentransfer benutzten viralen Vektoren, um das Potenzial für die klinische Anwendung voll ausschöpfen zu können.

\section{Korrespondenzadresse}

\section{T. A. Münch}

Retinal Circuits and Optogenetics, Centre for Integrative Neuroscience and Bernstein Center for Computational Neuroscience, Universität Tübingen

Otfried-Müller-Str. 25, 72076 Tübingen,

Deutschland

thomas.muench@cin.uni-tuebingen.de

\section{Einhaltung ethischer Richtlinien}

Interessenkonflikt. M. Mutter, B. Benkner und T.A. Münch geben an, dass kein Interessenkonflikt besteht. Unterstützung durch Mittel der Deutschen Forschungsgemeinschaft (DFG EXC 307) und der Tistou \& Charlotte Kerstan Stiftung.

Dieser Beitrag beinhaltet keine von den Autoren durchgeführten Studien an Menschen oder Tieren.

Open Access. Dieser Artikel wird unter der Creative Commons Namensnennung 4.0 International Lizenz (http://creativecommons.org/licenses/by/4.0/deed. de) veröffentlicht, welche die Nutzung, Vervielfältigung, Bearbeitung, Verbreitung und Wiedergabe in jeglichem Medium und Format erlaubt, sofern Sie den/die ursprünglichen Autor(en) und die Quelle ordnungsgemäß nennen, einen Linkzur Creative Commons Lizenz beifügen und angeben, ob Änderungen vorgenommen wurden.

\section{Literatur}

1. Bi A, Cui J, Ma YP et al (2006) Ectopic expression of a microbial-type rhodopsin restores visual responses in mice with photoreceptor degeneration. Neuron 50:23-33. doi:10.1016/j.neuron.2006.02. 026

2. Busskamp V, Duebel J, Balya D et al (2010) Genetic reactivation of cone photoreceptors restores visual responses in retinitis pigmentosa. Science 329:413-417. doi:10.1126/science.1190897

3. Byrne LC, Dalkara D, Luna G et al (2015) Viral-mediated RdCVF and RdCVFL expression protects cone and rod photoreceptors in retinal degeneration. J Clin Invest 125:105-116. doi:10. $1172 / \mathrm{JCl} 65654$

4. Cehajic-Kapetanovic J, Eleftheriou C, Allen AE et al (2015) Restoration of Vision with Ectopic Expression of Human Rod Opsin. Curr Biol 25:2111-2122. doi:10.1016/j.cub.2015.07.029

5. Editorial (2011) Method of the Year 2010. Nat Meth 8:1-1. doi:10.1038/nmeth.f.321

6. Gaub BM, Berry MH, Holt AE et al (2015) Optogenetic vision restoration using rhodopsin for enhanced sensitivity. Mol Ther 23:1562-1571. doi:10.1038/mt.2015.121

7. Gendron SP, Bastien N, Mallet JD et al (2013) The 3895-bp mitochondrialDNAdeletion in the human eye: a potential involvement in corneal ageing and macular degeneration. Mutagenesis 28:197-204. doi:10.1093/mutage/ges071

8. Kleinlogel S, Feldbauer K, Dempski RE et al (2011) Ultra light-sensitive and fast neuronal activation with the $\mathrm{Ca}(2)+-$ permeable channelrhodopsin CatCh. Nat Neurosci 14:513-518. doi:10.1038/nn 2776

9. Lagali PS, Balya D, Awatramani GB et al (2008) Light-activated channels targeted to ON bipolar cells restorevisual function in retinal degeneration. Nat Neurosci 11:667-675. doi:10.1038/nn.2117

10. MarcR,PfeifferR, Jones B (2014) Retinal prosthetics, optogenetics, and chemical photoswitches. ACS Chem Neurosci 5:895-901. doi:10.1021/ cn5001233

11. Nagel G, Szellas T, Huhn W et al (2003) Channelrhodopsin-2, a directly light-gated cation-selective membrane channel. Proc Natl Acad Sci USA 100:13940-13945. doi:10.1073/pnas.1936192100

12. Polosukhina A, Litt J, Tochitsky I et al (2012) Photochemical restoration of visual responses in blind mice. Neuron 75:271-282. doi:10.1016/j. neuron.2012.05.022

13. Sengupta A, Chaffiol A, Mace E et al (2016) Redshifted channelrhodopsin stimulation restores light responses in blind mice, macaque retina, and human retina. EMBO Mol Med 8:1248-1264. doi:10.15252/emmm.201505699

14. Stingl K, Bartz-Schmidt KU, Besch D et al (2013) Artificial vision with wirelessly powered subretinal electronic implant alpha-IMS. Proc Biol Sci 280:20130077. doi:10.1098/rspb.2013.0077

15. Tochitsky I, Polosukhina A, Degtyar VE et al (2014) Restoring visual function to blind mice with a photoswitch that exploits electrophysiological remodeling of retinal ganglion cells. Neuron 81:800-813. doi:10.1016/j.neuron.2014.01.003

16. van Wyk M, Pielecka-Fortuna J, Lowel S et al (2015) Restoring the ON switch in blind retinas:
Opto-mGluR6, a next-generation, cell-tailored optogenetic tool. PLOS Biol 13:e1002143. doi:10. 1371/journal.pbio.1002143

17. Wu J, Seregard S, Algvere PV (2006) Photochemical damage of the retina. Surv Ophthalmol 51:461-481. doi:10.1016/j.survophthal.2006.06. 009

18. Yue L, Weiland JD, Roska B et al (2016) Retinal stimulation strategies to restore vision: fundamentals and systems. Prog Retin Eye Res 53:21-47. doi:10.1016/j.preteyeres.2016.05.002 
Hier steht eine Anzeige.

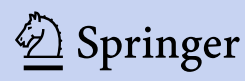

